\title{
Recoil-Induced-Resonances in Nonlinear, Ground-State, Pump-Probe Spectroscopy
}

\author{
C. P. Search and P. R. Berman \\ Physics Department, University of Michigan, Ann Arbor, MI, 48109-1120
}

(November 5, 2018)

Typeset using REVTEX 


\begin{abstract}
A theory of pump-probe spectroscopy is developed in which optical fields drive two-photon Raman transitions between ground states of an ensemble of three-level atoms. Effects related to the recoil the atoms undergo as a result of their interactions with the fields are fully accounted for in this theory. The linear absorption coefficient of a weak probe field in the presence of two pump fields of arbitrary strength is calculated. For subrecoil cooled atoms, the spectrum consists of eight absorption lines and eight emission lines. In the limit that $\chi_{1} \ll \chi_{2}$, where $\chi_{1}$ and $\chi_{2}$ are the Rabi frequencies of the two pump fields, one recovers the absorption spectrum for a probe field interacting with an effective two-level atom in the presence of a single pump field. However when $\chi_{1} \gtrsim \chi_{2}$, new interference effects arise that allow one to selectively turn on and off some of these recoil induced resonances.
\end{abstract}

\title{
I. INTRODUCTION
}

Recent advances in laser cooling, atom optics, and Bose-Einstein condensation have underlined the role played by atomic recoil in atom-field interactions. A measure of the importance of recoil effects is the recoil frequency, $\omega_{\hbar \mathbf{k}}=\hbar k^{2} / 2 M$, associated with the absorption, emission or scattering of radiation of wavelength $\lambda=2 \pi / k$ by an atom of mass $M$. Once this quantity becomes greater than or comparable to decay rates or Doppler widths that characterize the spectral response of atoms, recoil can lead to new features in absorption or emission line shapes. One class of such phenomena has been termed recoilinduced resonances (RIR) [1-8], which occur when a weak probe and strong pump field simultaneously drive a given atomic transition. Interesting in their own right, the RIR have been used to determine the velocity distribution of laser-cooled atoms [7], as a probe of Bose-Einstein condensates [9] [10], and in a feedback mechanism in stochastic cooling [1]. Related to the RIR is the so-called collective atomic recoil laser (CARL), which operates on 
similar principles but in a somewhat different parameter range [12]. Both the RIR and CARL represent new diagnostic probes of cold-atom sysytems. Recently, Moore and Meystre [13] proposed that CARL be used to entangle optical and matter fields, as well as to entangle different modes of the condensate excited by the optical fields. Our discussion is limited to situations in which the collective effects associated with CARL can be neglected. In this paper, we combine RIR with ground state spectroscopy 14 to obtain qualitatively new features in the probe absorption spectrum.

The scheme we adopt is based on the model developed in [14] involving Raman transitions, but for which all effects associated with atomic recoil were ignored. In that work a new type of interference was discovered, allowing one to selectively turn on and off certain lines in the absorption-emission spectrum by controlling the ratio of the Rabi frequencies of the two fields that comprise the two-photon pump field. Interference in a dressed state basis occurs between pathways involving the probe field and each of the two pump fields. Since the two pump fields impart different recoil momenta to the atoms, it is not at all obvious that the interference persists when recoil splittings are resolved. Part of the motivation for our calculation is to examine this question. In addition, we show that the interference persists even if the pump fields are in quantized, Fock states.

The probe absorption spectrum consists of as many as eight absorption-emission doublets which are fully resolvable if $\omega_{k}>\gamma$, where $\gamma$ is some effective ground state lifetime. This is in contrast to the RIR spectrum on dipole allowed optical transitions [1], where at most one absorption emission doublet is resolvable if $\omega_{k}<\gamma_{e}$, where $\gamma_{e}$ is an excited state decay rate. One might question the need to increase the number of recoil doublets in the probe spectrum, since a single doublet can be used to probe recoil effects. We show that the additional recoil structure reflects interesting quantum dynamics of the combined atom-field system, as well as providing some new applications.

In Sect. II, a model is developed for the interaction of the atoms with the pump fields; dressed states of the atom plus pump fields are defined. In Sect. III, the interaction with the probe field is introduced and the dressed state picture is used to obtain the probe absorption 
spectrum in the secular limit. In Sect. IV we discuss the results and possible applications. Nonsecular contributions to the absorption coefficient are calculated in an Appendix.

\section{EQUATIONS OF MOTION}

The atom-field system is indicated schematically in Fig.1. Ground state levels $\mid 1>$ and |2> are pumped incoherently with rates $\Lambda_{1}(\mathbf{p})$ and $\Lambda_{2}(\mathbf{p})$, respectively, and the population of both states decay with rate $\gamma$. If states $\mid 1>$ and $\mid 2>$ represent stable ground states of the atom, then the pumping rates and decay rate constitute a simple model for atoms that enter and leave the interaction volume. The ground to excited state transition frequencies are denoted by $\omega_{e j}(j=1,2)$. The pump fields 1 and 2 , which constitute the two-photon pump field, are denoted by the coupling constants $g_{1}$ and $g_{2}$, respectively. Pump field 1 couples only state $\mid 1>$ and excited state $\mid e>$, while pump 2 couples only states $\mid 2>$ and $\mid e>$. The pump fields have frequencies $\Omega_{1}$ and $\Omega_{2}$, and propagation vectors $\mathbf{k}_{1}$ and $\mathbf{k}_{2}$, respectively. In this section, equations are derived for the atom-pump field interaction, neglecting the incoherent pumping and decay of the ground state levels. In the following section, the interaction of the atoms with the probe field, $E_{p}$, which couples only states $\mid 1>$ and $|e\rangle$, is incorporated into the calculation, as are the incoherent pumping rates $\Lambda_{1}(\mathbf{p})$ and $\Lambda_{2}(\mathbf{p})$, and the ground state decay rate $\gamma$.

In contrast to [14] but as in [3], we use a quantized description of the pump fields. If the pump field detunings,

$$
\Delta_{2}=\Omega_{2}-\omega_{e 2} \approx \Delta_{1}=\Omega_{1}-\omega_{e 1} \equiv \Delta,
$$

are sufficiently large such that $\left|\chi_{1,2} / \Delta\right|^{2} \ll 1$ and $\left|\gamma_{e} / \Delta\right| \ll 1$, where the $\chi_{1,2}$ are defined below and $\gamma_{e}$ is the excited state decay rate, it is possible to adiabatically eliminate the excited state to arrive at an effective Hamiltonian involving only states $\mid 1>$ and $\mid 2>$ which is of the form 


$$
\begin{aligned}
H & =H_{a}+H_{r}+H_{a r} ; \\
H_{a} & =\sum_{\mathbf{p}}\left[\left(\hbar \omega_{1}+\frac{\mathbf{p}^{2}}{2 M}\right)|1, \mathbf{p}><1, \mathbf{p}|+\left(\hbar \omega_{2}+\frac{\mathbf{p}^{2}}{2 M}\right)|2, \mathbf{p}><2, \mathbf{p}|\right] \\
H_{r} & =\hbar \Omega_{1} a_{1}^{\dagger} a_{1}+\hbar \Omega_{2} a_{2}^{\dagger} a_{2} ; \\
H_{a r} & =\sum_{\mathbf{p}}\left[\hbar \frac{g_{1} g_{2}^{*} e^{i \mathbf{k}_{12} \cdot \mathbf{R}}}{\Delta}|2, \mathbf{p}><1, \mathbf{p}| a_{2}^{\dagger} a_{1}+\hbar \frac{g_{2} g_{1}^{*} e^{i \mathbf{k}_{21} \cdot \mathbf{R}}}{\Delta}|1, \mathbf{p}><2, \mathbf{p}| a_{1}^{\dagger} a_{2}\right],
\end{aligned}
$$

where $H_{a}$ is the Hamiltonian for the atom in which the center-of-mass momentum $\mathbf{p}$ has been quantized using periodic boundary conditions in a volume $V$ (assuming that the atoms are free and not subject to some trapping potential), $H_{r}$ is the free field Hamiltonian for the two pump fields, $H_{a r}$ represents the interaction of the two-photon pump field with an atom, in the rotating-wave approximation, and

$$
\mathbf{k}_{i j}=\mathbf{k}_{i}-\mathbf{k}_{j}
$$

Bare states, $\mid j, \mathbf{p} ; n_{1}, n_{2}>$, are defined to be eigenstates of $H_{a}+H_{r}$, where $j=1,2$ labels the internal state of the atom and $n_{1}$ and $n_{2}$ are the number of photons in pump fields 1 and 2 , respectively. A term in the Hamiltonian corresponding to the light shifts of the ground state levels, $H_{l s}=\sum_{\mathbf{p}}\left[\hbar \frac{\left|g_{1}\right|^{2}}{\Delta}|1, \mathbf{p}><1, \mathbf{p}| a_{1}^{\dagger} a_{1}+\hbar \frac{\left|g_{2}\right|^{2}}{\Delta}|2, \mathbf{p}><2, \mathbf{p}| a_{2}^{\dagger} a_{2}\right]$, has been omitted in Eq. (3a); such light shifts can be included by a redefinition of the ground state frequencies, $\omega_{2}+\frac{\left|g_{2}\right|^{2}}{\Delta}\left\langle a_{2}^{\dagger} a_{2}\right\rangle \rightarrow \omega_{2}$ and $\omega_{1}+\frac{\left|g_{1}\right|^{2}}{\Delta}\left\langle a_{1}^{\dagger} a_{1}\right\rangle \rightarrow \omega_{1}$

The matrix elements of the operator $e^{i \mathbf{k} \cdot \mathbf{R}}$ in the momentum-state basis are

$$
<\mathbf{p}\left|e^{i \mathbf{k} \cdot \mathbf{R}}\right| \mathbf{p}^{\prime}>=<\mathbf{p} \mid \mathbf{p}^{\prime}+\hbar \mathbf{k}>=\delta_{\mathbf{p}, \mathbf{p}^{\prime}+\hbar \mathbf{k}}
$$

This allows one to rewrite the interaction term as

$$
H_{a r}=\sum_{\mathbf{p}}\left[\hbar \frac{g_{1} g_{2}^{*}}{\Delta}\left|2, \mathbf{p}+\hbar \mathbf{k}_{12}><1, \mathbf{p}\right| a_{2}^{\dagger} a_{1}+\hbar \frac{g_{2} g_{1}^{*}}{\Delta}\left|1, \mathbf{p}><2, \mathbf{p}+\hbar \mathbf{k}_{12}\right| a_{1}^{\dagger} a_{2}\right] .
$$

The Hamiltonian $H$ results in an infinite ladder of decoupled two state manifolds $\left(\mathbf{p}, n_{1}, n_{2}\right)$ involving the states $\mid 1, \mathbf{p} ; n_{1}, n_{2}>$ and $\mid 2, \mathbf{p}+\hbar \mathbf{k}_{12} ; n_{1}-1, n_{2}+1>$. The Hamiltonian for the manifold $\left(\mathbf{p}, n_{1}, n_{2}\right)$ is

$$
H\left(\mathbf{p}, n_{1}, n_{2}\right)=\varepsilon\left(\mathbf{p}, n_{1}, n_{2}\right) \mathbf{I}+\hbar\left(\begin{array}{cc}
-\tilde{\delta}(\mathbf{p}) / 2 & G^{*} \\
G & \tilde{\delta}(\mathbf{p}) / 2
\end{array}\right)
$$


where $\mathbf{I}$ is the identity matrix

$$
\begin{gathered}
G=\frac{\chi_{1}^{*} \chi_{2}}{\Delta} \equiv|G| e^{i \phi_{d}}, \\
\varepsilon\left(\mathbf{p}, n_{1}, n_{2}\right)=\hbar\left(n_{1} \Omega_{1}+n_{2} \Omega_{2}\right)+\frac{\hbar}{2}\left(\omega_{\mathbf{p}}+\omega_{\mathbf{p}+\hbar \mathbf{k}_{12}}+\omega_{1}+\omega_{2}+\Omega_{2}-\Omega_{1}\right), \\
\tilde{\delta}(\mathbf{p})=\delta_{12}-\omega_{\hbar \mathbf{k}_{12}}-\frac{\mathbf{p} \cdot \mathbf{k}_{12}}{M} ; \\
\delta_{12}=\Delta_{1}-\Delta_{2}=\left(\Omega_{1}-\Omega_{2}\right)-\omega_{21},
\end{gathered}
$$

$\hbar \omega_{\mathbf{p}}=\frac{\mathbf{p}^{2}}{2 M}$, and $\chi_{2}=g_{2} \sqrt{n_{2}+1}, \chi_{1}=g_{1} \sqrt{n_{1}}$.

The dressed states are defined to be the eigenstates of the matrix in Eq.(7), with energies

$$
\begin{aligned}
& E_{B, A}(\mathbf{p})=\varepsilon\left(\mathbf{p}, n_{1}, n_{2}\right) \pm \frac{\hbar \omega_{A B}(\mathbf{p})}{2} \\
& \omega_{A B}(\mathbf{p})=\sqrt{4|G|^{2}+\tilde{\delta}(\mathbf{p})^{2}}
\end{aligned}
$$

and associated eigenkets 15

$$
\left(\begin{array}{l}
\mid A_{0}> \\
\mid B_{0}>
\end{array}\right)=\mathbf{T}^{*}(\mathbf{p})\left(\begin{array}{c}
\mid 2, \mathbf{p}+\hbar \mathbf{k}_{12} ; n_{1}-1, n_{2}+1> \\
\mid 1, \mathbf{p} ; n_{1}, n_{2}>
\end{array}\right)
$$

where

$$
\mathbf{T}(\mathbf{p})=\left(\begin{array}{cc}
e^{i \phi_{d} / 2} \cos [\theta(\mathbf{p})] & -e^{-i \phi_{d} / 2} \sin [\theta(\mathbf{p})] \\
e^{i \phi_{d} / 2} \sin [\theta(\mathbf{p})] & e^{-i \phi_{d} / 2} \cos [\theta(\mathbf{p})]
\end{array}\right)
$$

and

$$
\cos [\theta(\mathbf{p})]=\left[\frac{1}{2}\left(1+\frac{\tilde{\delta}(\mathbf{p})}{\omega_{A B}(\mathbf{p})}\right)\right]^{1 / 2} .
$$

The value of $\theta(\mathbf{p})$ is restricted to the range $0 \leq \theta(\mathbf{p}) \leq \pi / 4$ for $\delta(\mathbf{p}) \geq 0$ and $\pi / 4 \leq \theta(\mathbf{p}) \leq$ $\pi / 2$ for $\tilde{\delta}(\mathbf{p}) \leq 0$. For $\theta(\mathbf{p}) \sim 0(\tilde{\delta}(\mathbf{p})>0$ and $\tilde{\delta}(\mathbf{p}) /|G| \gg 1),\left|A_{0}>\sim\right| 2, \mathbf{p}+\hbar \mathbf{k}_{12} ; n_{1}-1, n_{2}+$ $1>$ while for $\theta(\mathbf{p}) \sim \pi / 2(\tilde{\delta}(\mathbf{p})<0$ and $|\tilde{\delta}(\mathbf{p})| /|G| \gg 1),\left|B_{0}>\sim\right| 2, \mathbf{p}+\hbar \mathbf{k}_{12} ; n_{1}-1, n_{2}+1>$. 


\section{PROBE FIELD ABSORPTION IN THE SECULAR LIMIT}

The effect of the probe field is to induce transitions between states in different manifolds. As is customary in dressed atom approaches, the probe is treated as a classical field,

$$
\mathbf{E}(\mathbf{R}, t)=\frac{1}{2} \widehat{\epsilon} E_{p} e^{i\left(\mathbf{k}_{p} \cdot \mathbf{R}-\Omega_{p} t\right)}+\text { c.c. },
$$

where $\widehat{\epsilon}$ is a unit polarization vector. For our problem, however, this choice represents a hybrid approach, since two-quantum processes involving the probe field and either of the pump fields mix classical and quantized fields. Although the probe field is treated classically, its effect on the momentum of the states must be accounted for explicitly. If the probe field detuning on the $1 \rightarrow e$ transition is sufficiently large to be consistent with the adiabatic elimination of the excited state, all transitions involving the probe occur via two-quantum transitions involving the probe field and either of the pump fields. In the bare state basis, starting from the $\left(\mathbf{p}, n_{1}, n_{2}\right)$ manifold, probe field absorption corresponds to transitions $\left|1, \mathbf{p} ; n_{1}, n_{2}>\rightarrow\right| 1, \mathbf{p}+\hbar \mathbf{k}_{p 1} ; n_{1}+1, n_{2}>$ or $\left|1, \mathbf{p} ; n_{1}, n_{2}>\rightarrow\right| 2, \mathbf{p}+\hbar \mathbf{k}_{p 2} ; n_{1}, n_{2}+1>$, where the second photon is emitted into either the pump 1 or pump 2 modes, respectively. Similarly, probe gain corresponds to transitions $\left|1, \mathbf{p} ; n_{1}, n_{2}>\rightarrow\right| 1, \mathbf{p}-\hbar \mathbf{k}_{p 1} ; n_{1}-1, n_{2}>$ or $\left|2, \mathbf{p}+\hbar \mathbf{k}_{12} ; n_{1}-1, n_{2}+1>\rightarrow\right| 1, \mathbf{p}-\hbar \mathbf{k}_{p 1} ; n_{1}-1, n_{2}>$. This is illustrated in Fig. 2(a).

This picture of probe field absorption or emission allows us to reach an important conclusion concerning interference between pathways involving both pump fields. The two absorption processes shown in Fig. 2(a) involve different final states and do not interfere, reinforcing the possibility mentioned in the Introduction that interference may be suppressed when recoil is taken into account. However, both final states belong to the same manifold the $\left(\mathbf{p}+\hbar \mathbf{k}_{p 1}, n_{1}+1, n_{2}\right)$ manifold. As such, when these states are dressed by the two-photon pump field, each state in the final state manifold will be coupled to the initial state by two separate pathways involving the probe field and each of the pump fields. This implies that terms in the probe absorption depending on the simultaneous presence of both pump fields will exhibit interference effects. In the case of probe gain, it is the initial states that differ, but the overall conclusion remains unchanged. 
The dressed state approach provides a convenient and relatively easy method for obtaining the probe absorption spectrum in the secular limit, where the frequency separation of the dressed states in a given doublet is much larger than the ground state decay rate $\gamma$. [It is assumed from this point onward that $\gamma_{e}\left(\chi_{1,2} / \Delta\right)^{2} \ll \gamma$, implying that the dressed states decay with rate $\gamma[3]]$. One need calculate only the dressed state energies and the transition matrix elements to obtain the spectrum. A more detailed treatment of the problem, allowing one to calculate non-secular contributions, is presented in Appendix B.

It is straightforward to generalize the dressed states defined in the previous section to include the two manifolds coupled to the initial manifold by the probe. The 0 , 1, and 2 manifolds refer to $\left(\mathbf{p}, n_{1}, n_{2}\right)=\left\{\left|1, \mathbf{p} ; n_{1}, n_{2}>,\right| 2, \mathbf{p}+\hbar \mathbf{k}_{12} ; n_{1}-1, n_{2}+1>\right\}$, $\left(\mathbf{p}+\hbar \mathbf{k}_{p 1}, n_{1}+1, n_{2}\right)=\left\{\left|1, \mathbf{p}+\hbar \mathbf{k}_{p 1} ; n_{1}+1, n_{2}>,\right| 2, \mathbf{p}+\hbar\left(\mathbf{k}_{12}+\mathbf{k}_{p 1}\right) ; n_{1}, n_{2}+1>\right\}$, and $\left(\mathbf{p}-\hbar \mathbf{k}_{p 1}, n_{1}-1, n_{2}\right)=\left\{\left|1, \mathbf{p}-\hbar \mathbf{k}_{p 1} ; n_{1}-1, n_{2}>,\right| 2, \mathbf{p}+\hbar\left(\mathbf{k}_{12}-\mathbf{k}_{p 1}\right) ; n_{1}-2, n_{2}+1>\right\}$, respectively. Taking the central energy of the initial manifold $\left(\mathbf{p}, n_{1}, n_{2}\right)$ arbitrarily equal to zero, one finds that the dressed state energies are given by

$$
\begin{aligned}
& E_{A, B}^{(0)}= \pm \frac{1}{2} \hbar \omega_{A B}^{(0)}(\mathbf{p}) \\
& E_{A, B}^{(1)}=\hbar \omega_{10}(\mathbf{p}) \pm \frac{1}{2} \hbar \omega_{A B}^{(1)}(\mathbf{p}) \\
& E_{A, B}^{(2)}=\hbar \omega_{20}(\mathbf{p}) \pm \frac{1}{2} \hbar \omega_{A B}^{(2)}(\mathbf{p}),
\end{aligned}
$$

where

$$
\begin{gathered}
\omega_{A B}^{(i)}(\mathbf{p})=\sqrt{4|G|^{2}+\delta_{i}(\mathbf{p})^{2}}, \\
\delta_{0}(\mathbf{p})=\tilde{\delta}(\mathbf{p})=\delta_{12}-\omega_{\hbar \mathbf{k}_{12}}-\frac{\mathbf{p} \cdot \mathbf{k}_{12}}{M} ; \\
\delta_{1}(\mathbf{p})=\tilde{\delta}\left(\mathbf{p}+\hbar \mathbf{k}_{p 1}\right)=\delta_{12}-\omega_{\hbar \mathbf{k}_{12}}-\frac{\left(\mathbf{p}+\hbar \mathbf{k}_{p 1}\right) \cdot \mathbf{k}_{12}}{M} ; \\
\delta_{2}(\mathbf{p})=\tilde{\delta}\left(\mathbf{p}-\hbar \mathbf{k}_{p 1}\right)=\delta_{12}-\omega_{\hbar \mathbf{k}_{12}}-\frac{\left(\mathbf{p}-\hbar \mathbf{k}_{p 1}\right) \cdot \mathbf{k}_{12}}{M}, \\
\hbar \omega_{10}(\mathbf{p})=\varepsilon\left(\mathbf{p}+\hbar \mathbf{k}_{p 1}, n_{1}+1, n_{2}\right)-\varepsilon\left(\mathbf{p}, n_{1}, n_{2}\right)=\hbar\left(\Omega_{1}+\omega_{\hbar \mathbf{k}_{p 1}}+\frac{\mathbf{k}_{p 1} \cdot \mathbf{p}}{M}+\frac{\hbar \mathbf{k}_{p 1} \cdot \mathbf{k}_{12}}{2 M}\right) ; \\
\hbar \omega_{20}(\mathbf{p})=\varepsilon\left(\mathbf{p}-\hbar \mathbf{k}_{p 1}, n_{1}-1, n_{2}\right)-\varepsilon\left(\mathbf{p}, n_{1}, n_{2}\right)=\hbar\left(-\Omega_{1}+\omega_{\hbar \mathbf{k}_{p 1}}-\frac{\mathbf{k}_{p 1} \cdot \mathbf{p}}{M}-\frac{\hbar \mathbf{k}_{p 1} \cdot \mathbf{k}_{12}}{2 M}\right),
\end{gathered}
$$


and it has also been assumed that $n_{1}, n_{2} \gg 1$ such that $G^{(0)}=g_{1}^{*} g_{2} \sqrt{n_{1}\left(n_{2}+1\right)} / \Delta \approx$ $G^{(1)}=g_{1}^{*} g_{2} \sqrt{\left(n_{1}+1\right)\left(n_{2}+1\right)} / \Delta \approx G^{(2)}=g_{1}^{*} g_{2} \sqrt{\left(n_{1}-1\right)\left(n_{2}+1\right)} / \Delta \equiv G$. The dressed state angles are given by

$$
\cos \left[\theta_{i}(\mathbf{p})\right]=\left[\frac{1}{2}\left(1+\frac{\delta_{i}(\mathbf{p})}{\omega_{A B}^{(i)}(\mathbf{p})}\right)\right]^{1 / 2}
$$

and dressed state kets are defined by

$$
\left(\begin{array}{c}
\mid A_{i}> \\
\mid B_{i}>
\end{array}\right)=\mathbf{T}_{i}^{*}(\mathbf{p})\left(\begin{array}{c}
\mid 2, \mathbf{p}+\hbar\left[\mathbf{k}_{12}-(-1)^{i}\left(1-\delta_{i, 0}\right) \mathbf{k}_{p 1}\right] ; n_{1}-1-(-1)^{i}\left(1-\delta_{i, 0}\right), n_{2}+1> \\
\mid 1, \mathbf{p}-(-1)^{i}\left(1-\delta_{i, 0}\right) \hbar \mathbf{k}_{p 1} ; n_{1}-(-1)^{i}\left(1-\delta_{i, 0}\right), n_{2}>
\end{array}\right)
$$

where

$$
\mathbf{T}_{i}(\mathbf{p})=\left(\begin{array}{cc}
e^{i \phi_{d} / 2} \cos \left[\theta_{i}(\mathbf{p})\right] & -e^{-i \phi_{d} / 2} \sin \left[\theta_{i}(\mathbf{p})\right] \\
e^{i \phi_{d} / 2} \sin \left[\theta_{i}(\mathbf{p})\right] & e^{-i \phi_{d} / 2} \cos \left[\theta_{i}(\mathbf{p})\right]
\end{array}\right)
$$

The absorption coefficient, $\alpha$, is proportional to the rate at which energy is absorbed from $(\alpha>0)$ or emitted into $(\alpha<0)$ the probe field. Absorption corresponds to transitions from initial dressed states $I=A_{0}, B_{0}$ to final state dressed states $J=A_{1}, B_{1}$, while emission corresponds to transitions from initial dressed states $I=A_{0}, B_{0}$ to final state dressed states $J=A_{2}, B_{2}$. For a given transition, the contribution to the absorption coefficient is proportional to $N V \hbar \Omega_{p} \gamma \rho_{J J} /\left|E_{p}\right|^{2}$, where $N$ is the atom density and $\rho_{J J}$ is the steady state population in state $J$ owing to the $I \rightarrow J$ transition. The final state population $\rho_{J J}(\mathbf{p})$ is equal to $\left[\Lambda_{I}(\mathbf{p}) / \gamma\right]|<J| V_{p}|I>|^{2} L_{I J}$, where $\Lambda_{I}(\mathbf{p})$ is the pumping rate for initial dressed state $I$ [16], $<J\left|V_{p}\right| I>$ is a matrix element for the $I-J$ transition and $L_{I J}$ is a Lorentzian having width $\gamma$, centered at the $I-J$ transition frequency. The transition frequencies $\Delta_{I J}(\mathbf{p})$ may be directly read from Fig. 2(b) or obtained from Eq. (14). Consequently, it is necessary to specify $\Lambda_{I}(\mathbf{p})$ and to calculate the transition matrix elements $\left\langle J\left|V_{p}\right| I\right\rangle$ in order to arrive at an expression for the probe absorption.

In the bare state representation, the pumping matrix for the intitial state manifold is taken to be of the form 


$$
\Lambda(\mathbf{p})=\left(\begin{array}{cc}
\dot{\rho}_{11}(\mathbf{p}) & 0 \\
0 & \dot{\rho}_{22}\left(\mathbf{p}+\hbar \mathbf{k}_{12}\right)
\end{array}\right)_{p u m p}=\left(\begin{array}{cc}
\Lambda_{1}(\mathbf{p}) & 0 \\
0 & \Lambda_{2}\left(\mathbf{p}+\hbar \mathbf{k}_{\mathbf{1 2}}\right)
\end{array}\right)
$$

which, when converted to the dressed state basis becomes

$$
\begin{aligned}
& \Lambda_{d}(\mathbf{p})=\left(\begin{array}{cc}
\Lambda_{A}(\mathbf{p}) & \Lambda_{A B}(\mathbf{p}) \\
\Lambda_{A B}(\mathbf{p}) & \Lambda_{B}(\mathbf{p})
\end{array}\right) \\
& =\left(\begin{array}{cc}
\Lambda_{2}\left(\mathbf{p}+\hbar \mathbf{k}_{12}\right) \cos ^{2}\left[\theta_{0}(\mathbf{p})\right]+\Lambda_{1}(\mathbf{p}) \sin ^{2}\left[\theta_{0}(\mathbf{p})\right] & \frac{1}{2}\left[\Lambda_{2}\left(\mathbf{p}+\hbar \mathbf{k}_{12}\right)-\Lambda_{1}(\mathbf{p})\right] \sin \left[2 \theta_{0}(\mathbf{p})\right] \\
\frac{1}{2}\left[\Lambda_{2}\left(\mathbf{p}+\hbar \mathbf{k}_{12}\right)-\Lambda_{1}(\mathbf{p})\right] \sin \left[2 \theta_{0}(\mathbf{p})\right] & \Lambda_{1}(\mathbf{p}) \cos ^{2}\left[\theta_{0}(\mathbf{p})\right]+\Lambda_{2}\left(\mathbf{p}+\hbar \mathbf{k}_{12}\right) \sin ^{2}\left[\theta_{0}(\mathbf{p})\right]
\end{array}\right)
\end{aligned}
$$

In the secular limit, the off-diagonal pumping terms can be neglected since they give rise to terms of order $\gamma / \omega_{A B}^{(i)}(\mathbf{p}) \ll 1$. Moreover, for the present we will set $\Lambda_{2}\left(\mathbf{p}+\hbar \mathbf{k}_{12}\right)=0$ and generalize the results to nonvanishing $\Lambda_{2}\left(\mathbf{p}+\hbar \mathbf{k}_{12}\right)$ in the next section. Thus, we take a pumping matrix of the form

$$
\Lambda_{d}(\mathbf{p})=\left(\begin{array}{cc}
\Lambda_{1}(\mathbf{p}) \sin ^{2}\left[\theta_{0}(\mathbf{p})\right] & 0 \\
0 & \Lambda_{1}(\mathbf{p}) \cos ^{2}\left[\theta_{0}(\mathbf{p})\right]
\end{array}\right) .
$$

We now turn our attention to the transition matrix element $<J\left|V_{p}\right| I>$. As an example consider $<A_{1}\left|V_{p}\right| A_{0}>$, which is the amplitude for the transition $\left|A_{0}>\rightarrow\right| A_{1}>$ involving probe absorption and pump 1 or pump 2 emission. This transition is illustrated in Fig. 2(c). The probe couples only to the $\left|1, \mathbf{p} ; n_{1}, n_{2}\right\rangle$ part of $\left|A_{0}\right\rangle$, leading to a factor $-e^{i \phi_{d} / 2} \sin \left[\theta_{0}(\mathbf{p})\right]$. The absorption of the probe is followed by emission into pump 2 taking the atom to the $\mid 2, \mathbf{p}+\hbar\left(\mathbf{k}_{12}+\mathbf{k}_{p 1}\right) ; n_{1}, n_{2}+1>$ component of $\mid A_{1}>$ and leading to a factor $e^{-i \phi_{d} / 2} \cos \left[\theta_{1}(\mathbf{p})\right]$ or emission into pump 1 taking the atom to the the $\mid 1, \mathbf{p}+\hbar \mathbf{k}_{p 1} ; n_{1}+1, n_{2}>$ component and leading to a factor $-e^{i \phi_{d} / 2} \sin \left[\theta_{1}(\mathbf{p})\right]$. The coupling strengths for these two, two-photon transitions are $G_{1}^{*}$ and $G_{2}^{*}$, respectively, where

$$
G_{1}=\frac{\chi_{p}^{*} \chi_{1}}{\Delta} ; \quad G_{2}=\frac{\chi_{p}^{*} \chi_{2}}{\Delta}
$$

The two processes add coherently and one finds

$$
<A_{1}|V| A_{0}>=\hbar\left\{G_{2}^{*} e^{i \phi_{d}} \cos \left[\theta_{1}(\mathbf{p})\right]-G_{1}^{*} \sin \left[\theta_{1}(\mathbf{p})\right]\right\}\left\{-\sin \left[\theta_{0}(\mathbf{p})\right]\right\} e^{-i \Omega_{p} t}
$$


Other matrix elements are calculated in a similar manner. For probe gain, pump fields 1 and 2 couple to the $\mid 1, \mathbf{p} ; n_{1}, n_{2}>$ and $\mid 2, \mathbf{p}+\hbar \mathbf{k}_{12} ; n_{1}-1, n_{2}+1>$ components of the dressed states in the 0 manifold, respectively, while the probe field couples to the $\mid 1, \mathbf{p}-\hbar \mathbf{k}_{p 1} ; n_{1}-1, n_{2}>$ component of the dressed states in the 2 manifold. Explicit expressions for the matrix elements are given in Appendix A.

Combining all transitions and summing over $\mathbf{p}$, one finds an absorption coefficient proportional to

$\alpha \propto \frac{\gamma^{2}}{\left|\chi_{p}\right|^{2}} \sum_{\mathbf{p}} \sum_{I=\left\{A_{o}, B_{o}\right\}} \frac{\Lambda_{I}(\mathbf{p})}{\gamma}\left(\sum_{J=\left\{A_{1}, B_{1}\right\}} \frac{|<J| V_{p}|I>|^{2}}{\left[\Omega_{p}-\Delta_{I J}(\mathbf{p})\right]^{2}+\gamma^{2}}-\sum_{J=\left\{A_{2}, B_{2}\right\}} \frac{|<J| V_{p}|I>|^{2}}{\left[\Omega_{p}-\Delta_{I J}(\mathbf{p})\right]^{2}+\gamma^{2}}\right)$

For a sub-recoiled cooled vapor, we can set $\Lambda_{1}(\mathbf{p})=\Lambda_{1} \delta_{\mathbf{p}, \mathbf{0}}$, such that $\Lambda_{A}(\mathbf{p})=\Lambda_{A} \delta_{\mathbf{p}, \mathbf{0}}$ and $\Lambda_{B}(\mathbf{p})=\Lambda_{B} \delta_{\mathbf{p}, \mathbf{0}}$, where

$$
\Lambda_{A}=\Lambda_{1} \sin ^{2} \theta_{0} ; \quad \Lambda_{B}=\Lambda_{1} \cos ^{2} \theta_{0},
$$

$\theta_{i} \equiv \theta_{i}(\mathbf{0})\left[\right.$ and, for future reference, $\Delta_{I J} \equiv \Delta_{I J}(\mathbf{0}), \omega_{A B}^{(i)} \equiv \omega_{A B}^{(i)}(\mathbf{0})$, etc. $]$ and $\delta_{\mathbf{p}, \mathbf{0}}$ is a kronecker delta. In this limit, one finds the absorption coefficient in the secular limit to be 


$$
\begin{aligned}
& \left(\frac{\alpha}{\alpha_{0}}\right)_{\mathrm{sec}}=\frac{|G|}{|\Delta|}\left[\left(\psi \eta \sin \theta_{1}-\frac{1}{\eta} \cos \theta_{1}\right)^{2}\left(\frac{\Lambda_{A}}{\gamma} \sin ^{2} \theta_{0} L_{A_{0} A_{1}}\left(\Delta^{\prime}\right)+\frac{\Lambda_{B}}{\gamma} \cos ^{2} \theta_{0} L_{B_{0} A_{1}}\left(\Delta^{\prime}\right)\right)\right. \\
& +\left(\psi \eta \cos \theta_{1}+\frac{1}{\eta} \sin \theta_{1}\right)^{2}\left(\frac{\Lambda_{A}}{\gamma} \sin ^{2} \theta_{0} L_{A_{0} B_{1}}\left(\Delta^{\prime}\right)+\frac{\Lambda_{B}}{\gamma} \cos ^{2} \theta_{0} L_{B_{0} B_{1}}\left(\Delta^{\prime}\right)\right) \\
& -\left(\psi \eta \sin \theta_{0}-\frac{1}{\eta} \cos \theta_{0}\right)^{2} \frac{\Lambda_{A}}{\gamma}\left(\sin ^{2} \theta_{2} L_{A_{0} A_{2}}\left(\Delta^{\prime}\right)+\cos ^{2} \theta_{2} L_{A_{0} B_{2}}\left(\Delta^{\prime}\right)\right) \\
& \left.-\left(\psi \eta \cos \theta_{0}+\frac{1}{\eta} \sin \theta_{0}\right)^{2} \frac{\Lambda_{B}}{\gamma}\left(\sin ^{2} \theta_{2} L_{B_{0} A_{2}}\left(\Delta^{\prime}\right)+\cos ^{2} \theta_{2} L_{B_{0} B_{2}}\left(\Delta^{\prime}\right)\right)\right] ; \\
& L_{A_{0} A_{1}}\left(\Delta^{\prime}\right)=\frac{\gamma^{2}}{\left(\Delta^{\prime}-\omega_{\hbar k_{p 1}}-\frac{1}{2}\left(\omega_{A B}^{(0)}-\omega_{A B}^{(1)}\right)\right)^{2}+\gamma^{2}} ; \\
& L_{B_{0} A_{1}}\left(\Delta^{\prime}\right)=\frac{\gamma^{2}}{\left(\Delta^{\prime}-\omega_{\hbar k_{p 1}}+\frac{1}{2}\left(\omega_{A B}^{(0)}+\omega_{A B}^{(1)}\right)\right)^{2}+\gamma^{2}} ; \\
& L_{A_{0} B_{1}}\left(\Delta^{\prime}\right)=\frac{\gamma^{2}}{\left(\Delta^{\prime}-\omega_{\hbar k_{p 1}}-\frac{1}{2}\left(\omega_{A B}^{(0)}+\omega_{A B}^{(1)}\right)\right)^{2}+\gamma^{2}} ; \\
& L_{B_{0} B_{1}}\left(\Delta^{\prime}\right)=\frac{\gamma^{2}}{\left(\Delta^{\prime}-\omega_{\hbar k_{p 1}}+\frac{1}{2}\left(\omega_{A B}^{(0)}-\omega_{A B}^{(1)}\right)\right)^{2}+\gamma^{2}} ; \\
& L_{A_{0} A_{2}}\left(\Delta^{\prime}\right)=\frac{\gamma^{2}}{\left(\Delta^{\prime}+\omega_{\hbar \mathbf{k}_{p 1}}+\frac{1}{2}\left(\omega_{A B}^{(0)}-\omega_{A B}^{(2)}\right)\right)^{2}+\gamma^{2}} ; \\
& L_{A_{0} B_{2}}\left(\Delta^{\prime}\right)=\frac{\gamma^{2}}{\left(\Delta^{\prime}+\omega_{\hbar \mathbf{k}_{p 1}}+\frac{1}{2}\left(\omega_{A B}^{(0)}+\omega_{A B}^{(2)}\right)\right)^{2}+\gamma^{2}} ; \\
& L_{B_{0} A_{2}}\left(\Delta^{\prime}\right)=\frac{\gamma^{2}}{\left(\Delta^{\prime}+\omega_{\hbar \mathbf{k}_{p 1}}-\frac{1}{2}\left(\omega_{A B}^{(0)}+\omega_{A B}^{(2)}\right)\right)^{2}+\gamma^{2}} ; \\
& L_{B_{0} B_{2}}\left(\Delta^{\prime}\right)=\frac{\gamma^{2}}{\left(\Delta^{\prime}+\omega_{\hbar k_{p 1}}-\frac{1}{2}\left(\omega_{A B}^{(0)}-\omega_{A B}^{(2)}\right)\right)^{2}+\gamma^{2}} ;
\end{aligned}
$$

where

$$
\psi=\Delta /|\Delta|
$$

is the sign of the detuning for each of the three fields,

$$
\eta=\sqrt{\left|\chi_{1}\right| /\left|\chi_{2}\right|},
$$




$$
\begin{gathered}
\Delta^{\prime}=\delta_{p 1}-\frac{\hbar \mathbf{k}_{p 1} \cdot \mathbf{k}_{12}}{2 M} \\
\delta_{p 1}=\Omega_{p}-\Omega_{1} ; \quad \delta_{p 2}=\Omega_{p}-\Omega_{2}-\omega_{21}, \\
\alpha_{0}=\frac{k_{p} N d_{1 e}^{2}}{2 \hbar \epsilon_{0} \gamma}
\end{gathered}
$$

and $d_{1 e}$ is a bare state dipole moment matrix element for the $|1\rangle \rightarrow|e\rangle$ transition.

The first four resonances in $\left(\frac{\alpha}{\alpha_{0}}\right)_{\mathrm{sec}}$ correspond to probe absorption while the last four correspond to probe gain. The spectrum is shown in Fig. 3. The line widths of all the resonances equal $\gamma$; consequently, the recoil induced resonances in ground state spectroscopy are fully resolved if $\omega_{k}>\gamma$. The secular contribution does not vanish in the limit that $|\delta(\mathbf{p}=\mathbf{0}) / G| \ll 1$, even though $\Lambda_{A}=\Lambda_{B}$ in this limit. As long as the recoil frequency is larger than $\gamma$ and the Doppler width associated with the two-photon pump transition, the absorption and emission contributions to the probe response do not cancel one another.

The most significant feature of $\left(\frac{\alpha}{\alpha_{0}}\right)_{\text {sec }}$ is that the line strengths involve factors such as $\left(\psi \eta \sin \theta_{1}-\frac{1}{\eta} \cos \theta_{1}\right)^{2}$ which allows one to manipulate the strength of the lines by controlling the sign of the field detuning and the ratio of the pump field amplitudes. These factors are an indication of interference between the two "two-photon probe" fields which can both lead to absorption or gain in $E_{p}$. Because the two lines in the doublets have different strengths, one can adjust $\psi$ and $\eta$ to turn off one of the lines. For example, the absorption doublet, $L_{A_{0} A_{1}}$ and $L_{B_{0} B_{1}}$, consists of the lines at $\Delta^{\prime}=\omega_{\hbar \mathbf{k}_{p 1}}+\frac{1}{2}\left(\omega_{A B}^{(0)}-\omega_{A B}^{(1)}\right)$ and $\omega_{\hbar \mathbf{k}_{p 1}}-\frac{1}{2}\left(\omega_{A B}^{(0)}-\omega_{A B}^{(1)}\right)$ with strengths $\sim\left(\psi \eta \sin \theta_{1}-\frac{1}{\eta} \cos \theta_{1}\right)^{2}$ and $\sim\left(\psi \eta \cos \theta_{1}+\frac{1}{\eta} \sin \theta_{1}\right)^{2}$, respectively. Choosing $\psi=+1$ and $\eta^{2}=\cot \theta_{1}$ turns "off" the first line while $\psi=-1$ and $\eta^{2}=\tan \theta_{1}$ turns "off" the second line. This is shown in Fig. 4. When $|G|$ and $\delta_{12}$ are much larger than any of the recoil terms, $\theta_{0} \approx \theta_{1} \approx \theta_{2}$ and the emission lines are also turned "off". Consequently, by choosing $\psi=+1$ and $\eta^{2}=\cot \theta_{1}$ to turn off the $L_{A_{0} A_{1}}$ and $L_{B_{0} A_{1}}$ absorption lines, the $L_{A_{0} A_{2}}$ and $L_{A_{0} B_{2}}$ emission lines are also turned off.

A particularly interesting case occurs when $\mathbf{k}_{1} \approx \mathbf{k}_{2}$ so that $\omega_{A B}^{(0)}=\omega_{A B}^{(1)}=\omega_{A B}^{(2)}$ and $\theta_{0}=$ $\theta_{1}=\theta_{2}$. This would correspond to a two-photon pump field which imparts no momentum 
to the atoms so that the recoil splitting in the absorption spectrum can be attributed solely to the recoil due to the probe field acting with either of the pump fields, $\mathbf{k}_{p 2}=\mathbf{k}_{p 1}$. In this case, the line $L_{A_{0} A_{1}}\left(\Delta^{\prime}\right)$ is degenerate with $L_{B_{0} B_{1}}\left(\Delta^{\prime}\right)$ and $L_{A_{0} A_{2}}\left(\Delta^{\prime}\right)$ is degenerate with $L_{B_{0} B_{2}}\left(\Delta^{\prime}\right)$. Consequently, the spectrum consists of three absorption-emission doublets

centered at $\Delta^{\prime}=0,+\omega_{A B}^{(0)},-\omega_{A B}^{(0)}$. Moreover, the lines within each doublet are split by $2 \omega_{\hbar \mathbf{k}_{p 1}}$ which is independent of the strength or detuning of the pump fields.

When the effects of atomic recoil are neglected by setting all recoil momenta to zero in $\left(\frac{\alpha}{\alpha_{0}}\right)$, one obtains the same absorption spectrum given in [14. In the limit that $G_{1}=0$, one recovers a simple, recoil shifted Raman spectrum. In the limit that $G_{2}=0$, one recovers the central, secular components of the pump-probe spectrum associated with a single, twolevel optical transition [3]. In the limit that $\eta \ll 1$, while $G / \gamma \gg 1$ remains constant, the absorption spectrum mirrors that for the pump-probe spectrum associated with a two-level optical transition [3].

\section{DISCUSSION}

For a subrecoil cooled vapor, pumping to state 2 at a rate $\Lambda_{2}\left(\mathbf{p}+\hbar \mathbf{k}_{12}\right)=\Lambda_{2} \delta_{\mathbf{p}+\hbar \mathbf{k}_{12}, \mathbf{0}}$ doubles the number of absorption and emission lines in the probe spectrum, but does not result in any qualitatively new features. There will be an additional contribution to Eq. (52) in which $\theta_{i} \equiv \theta_{i}(\mathbf{0})$ is replaced by $\bar{\theta}_{i} \equiv \theta_{i}\left(-\hbar \mathbf{k}_{12}\right), \Delta_{I J}$ by $\bar{\Delta}_{I J} \equiv \Delta_{I J}\left(-\hbar \mathbf{k}_{12}\right), \omega_{A B}^{i}$ by $\bar{\omega}_{A B}^{i} \equiv \omega_{A B}^{i}\left(-\hbar \mathbf{k}_{12}\right), \Lambda_{A}$ by $\Lambda_{2} \cos ^{2} \bar{\theta}_{0}$ and $\Lambda_{B}$ by $\Lambda_{2} \sin ^{2} \bar{\theta}_{0}$. The absorption coefficient contains sixteen lines in all. The eight new spectral components display the same properties as the original eight but are displaced by an amount $\sim \frac{\hbar \mathbf{k}_{p 1} \cdot \mathbf{k}_{12}}{M}$. Figure 5 shows the secular absorption spectrum with all sixteen components when $\Lambda_{2}=\Lambda_{1}$. In the absence of recoil, the secular absorption coefficient vanishes when $\Lambda_{2}=\Lambda_{1}$ [14]. However, when recoil is included, the absorption and emission contributions to the probe response do not cancel one another when $\Lambda_{2}=\Lambda_{1}$, provided the recoil frequency is larger than $\gamma$ and the Doppler width associated with the two-photon pump transition. 
In Ref. [14] interference effects similar to those in Eq. (27a) were found. We have shown that the interference is independent of the field statistics and persisits even when recoil induced resonances are resolved.

The RIR offer several possibilities for applications. The existence of a number of tunable, well-resolved, gain peaks allows one to envision experiments in which lasing occurs at one or more of the gain positions. These peaks could be adjusted to coincide with modes of a ring cavity, for example. By sweeping the ratio of the two pump field intensities, one has a mechanism for modifying the probe gain or absorption, to the point of total suppression. The central frequency of the absorption-emission doublets can be controlled via pump field strength and detuning. For parallel pump fields, the frequency separation of the absorption emission doublets can be as large as $8 \omega_{k}$ and is independent of pump field strength and detuning. With line widths approaching $1 \mathrm{~Hz}$ or less, the probe spectrum can be used to measure the recoil frequency to a precision of order $10^{-7}$; this precision can be increased if the two-photon pump field is replaced by a pair of counterpropagating, two-photon pump fields [17]. The narrow resonances can also be used in schemes for obtaining "slow light" 18].

Pump-probe spectroscopy of Bose-Einstein condensates represents an interesting application of the ideas presented here. Bragg spectroscopy has recently been demonstrated in condensates [9] [10] as well as the stimulated generation of matter waves in a condensate by Rayleigh scattering [19. Pump-probe spectroscopy using electronic excited states would be unfeasible in condensates since there are no stable trapped condensates with electronic states which may be populated. Currently, the only multi-component condensates consist of two hyperfine states in ${ }^{87} R b$ [21] and the Zeeman states in the $F=1$ manifold of optically trapped $\mathrm{Na}$ [22]. Consequently, pump-probe spectroscopy would necessarily involve Raman transitions between stable ground states in the manner proposed here. The RIR spectrum of a weakly interacting Bose condensate should yield information about the spectrum of elementary excitations in the condensate which for small momenta have a linear dispersion relation while, for large momenta, have a quadratic dispersion similar to that of free atoms, 
but with a shift due to the mean-field interactions in the condensate. In addition, the linewidths of the RIR spectrum should be given by the zero point motion of the condensate in

the trapping potential provided $\left(\frac{\hbar}{m \Delta x}\right) k_{p 1,2}>\gamma$ and $\Delta x$ is the size of the condensate. However, the direct application of the results presented here to a condensate would be erroneous since a correct calculation of the RIR spectrum would have to account for the mean-field interactions between the atoms. This will be pursued in future work.

\section{ACKNOWLEDGMENTS}

C. P. S. and P. R. B. are pleased to acknowledge helpful discussions with B. Dubetsky. This research is supported by the National Science Foundation under Grant No. PHY9800981 and by the U. S. Army Research Office under Grant No. DAAG55-97-0113 and No. DAAD19-00-1-0412.

\section{APPENDIX A - MATRIX ELEMENTS OF V $I D$}

The interaction with the probe field may be expressed in terms of an effective two-photon interaction Hamiltonian similar to that in Eq. (3d),

$$
\mathbf{V}_{I}=\hbar \sum_{\mathbf{p}}\left(\frac{\chi_{p} g_{2}^{*}}{\Delta} e^{-i \Omega_{p} t}\left|2, \mathbf{p}+\hbar \mathbf{k}_{p 2}\right\rangle\left\langle 1, \mathbf{p}\left|a_{2}^{\dagger}+\frac{\chi_{p} g_{1}^{*}}{\Delta} e^{-i \Omega_{p} t}\right| 1, \mathbf{p}+\hbar \mathbf{k}_{p 1}\right\rangle\langle 1, \mathbf{p}| a_{1}^{\dagger}+\text { h.c. }\right)
$$

where $\chi_{p}=\frac{-1}{2 \hbar} d_{e 1} E_{p}$ is the Rabi frequency for the probe field and $d_{e 1}=\langle e|\mathbf{d} \cdot \widehat{\epsilon}| 1\rangle$ is a dipole matrix element. The matrix representation of $\mathbf{V}_{I}$ with respect to the bare state basis has the following nonvanishing elements

$$
\begin{aligned}
\left\langle 1, \mathbf{p} ; n_{1}, n_{2}\left|\mathbf{V}_{I}\right| 2, \mathbf{p}+\hbar \mathbf{k}_{12}+\hbar \mathbf{k}_{p 1} ; n_{1}, n_{2}+1\right\rangle & =\hbar G_{2} e^{i \Omega_{p} t} ; \\
\left\langle 1, \mathbf{p} ; n_{1}, n_{2}\left|\mathbf{V}_{I}\right| 1, \mathbf{p}+\hbar \mathbf{k}_{p 1} ; n_{1}+1, n_{2}\right\rangle & =\hbar G_{1} e^{i \Omega_{p} t} ; \\
\left\langle 1, \mathbf{p}-\hbar \mathbf{k}_{p 1} ; n_{1}-1, n_{2}\left|\mathbf{V}_{I}\right| 2, \mathbf{p}+\hbar \mathbf{k}_{12} ; n_{1}-1, n_{2}+1\right\rangle & =\hbar G_{2} e^{i \Omega_{p} t} ; \\
\left\langle 1, \mathbf{p}-\hbar \mathbf{k}_{p 1} ; n_{1}-1, n_{2}\left|\mathbf{V}_{I}\right| 1, \mathbf{p} ; n_{1}, n_{2}\right\rangle & =\hbar G_{1} e^{i \Omega_{p} t} ;
\end{aligned}
$$


and the hermitian conjugates of Eqs. (29). In Eqs. (29), $G_{1}$ and $G_{2}$ are two-photon probe Rabi frequencies defined as $G_{1}=\chi_{p}^{*} \chi_{1} / \Delta ; G_{2}=\chi_{p}^{*} \chi_{2} / \Delta$. Note that the coupling of the 1 and 2 manifolds to manifolds other than the 0 manifold has been ignored.

The matrix $\mathbf{V}_{I d}$ represents the interaction with the probe field in the dressed state basis and is defined as

$$
\mathbf{V}_{I d}=\mathbf{T V}_{I} \mathbf{T}^{\dagger}
$$

where $\mathbf{T}$ is given by the block diagonal matrix

$$
\mathbf{T}=\operatorname{diag}\left[\mathbf{T}_{1}(\mathbf{p}), \mathbf{T}_{0}(\mathbf{p}), \mathbf{T}_{2}(\mathbf{p})\right]
$$

The Rabi frequencies $\chi_{p}, \chi_{1}$, and $\chi_{2}$ may be expressed as

$$
\chi_{p}=\left|\chi_{p}\right| e^{i \phi} ; \chi_{1}=\left|\chi_{1}\right| e^{i \phi_{1}} ; \chi_{2}=\left|\chi_{2}\right| e^{i \phi_{2}}
$$

so that $\phi_{d}=\phi_{2}-\phi_{1}+\frac{\pi}{2}(1-\psi)$ since $G=|G| e^{i \phi_{d}}$. The matrix elements are:

$$
\begin{aligned}
& <A_{1}\left|\mathbf{V}_{I}\right| A_{0}>=\hbar e^{i\left(\phi-\phi_{1}\right)} e^{-i \Omega_{p} t} \sin \theta_{0}\left(-\left|G_{2}\right| \cos \theta_{1}+\psi\left|G_{1}\right| \sin \theta_{1}\right) ; \\
& <A_{1}\left|\mathbf{V}_{I}\right| B_{0}>=\hbar e^{i\left(\phi-\phi_{1}\right)} e^{-i \Omega_{p} t} \cos \theta_{0}\left(\left|G_{2}\right| \cos \theta_{1}-\psi\left|G_{1}\right| \sin \theta_{1}\right) ; \\
& <B_{1}\left|\mathbf{V}_{I}\right| A_{0}>=\hbar e^{i\left(\phi-\phi_{1}\right)} e^{-i \Omega_{p} t} \sin \theta_{0}\left(-\psi\left|G_{1}\right| \cos \theta_{1}-\left|G_{2}\right| \sin \theta_{1}\right) ; \\
& <B_{1}\left|\mathbf{V}_{I}\right| B_{0}>=\hbar e^{i\left(\phi-\phi_{1}\right)} e^{-i \Omega_{p} t} \cos \theta_{0}\left(\psi\left|G_{1}\right| \cos \theta_{1}+\left|G_{2}\right| \sin \theta_{1}\right) ; \\
& <A_{0}\left|\mathbf{V}_{I}\right| A_{2}>=\hbar e^{i\left(\phi-\phi_{1}\right)} e^{-i \Omega_{p} t} \sin \theta_{2}\left(-\left|G_{2}\right| \cos \theta_{0}+\psi\left|G_{1}\right| \sin \theta_{0}\right) ; \\
& <A_{0}\left|\mathbf{V}_{I}\right| B_{2}>=\hbar e^{i\left(\phi-\phi_{1}\right)} e^{-i \Omega_{p} t} \cos \theta_{2}\left(\left|G_{2}\right| \cos \theta_{0}-\psi\left|G_{1}\right| \sin \theta_{0}\right) ; \\
& <B_{0}\left|\mathbf{V}_{I}\right| A_{2}>=\hbar e^{i\left(\phi-\phi_{1}\right)} e^{-i \Omega_{p} t} \sin \theta_{2}\left(-\psi\left|G_{1}\right| \cos \theta_{0}-\left|G_{2}\right| \sin \theta_{0}\right) ; \\
& <B_{0}\left|\mathbf{V}_{I}\right| B_{2}>=\hbar e^{i\left(\phi-\phi_{1}\right)} e^{-i \Omega_{p} t} \cos \theta_{2}\left(\psi\left|G_{1}\right| \cos \theta_{0}+\left|G_{2}\right| \sin \theta_{0}\right) .
\end{aligned}
$$

The other elements follow from the hermiticity of $\mathbf{V}_{I d}$.

\section{APPENDIX B}

In this Appendix the absorption coefficient for the probe field is calculated, without making the secular approximation. The absorption coefficient, $\alpha$, and index change, $\Delta n$, 
arise from the imaginary and real parts of the macroscopic polarization in the Maxwell-Bloch equations for the probe field. They are given by the expressions

$$
\begin{aligned}
\alpha & =\alpha_{0} \operatorname{Im}\left(\frac{\gamma \rho_{1 e}^{\prime}}{\chi_{p}^{*}} V\right) ; \\
\Delta n & =-\alpha_{0} k_{p}^{-1} \operatorname{Re}\left(\frac{\gamma \rho_{1 e}^{\prime}}{\chi_{p}^{*}} V\right) ; \\
\alpha_{0} & =\frac{k_{p} N d_{1 e}^{2}}{2 \hbar \epsilon_{0} \gamma}
\end{aligned}
$$

where $V$ is the volume and $\rho_{1 e}^{\prime}$ is the part of the bare state density matrix element $\rho_{1 e}(\mathbf{R}, t)$ which is proportional to $e^{-i\left(\mathbf{k}_{p} \cdot \mathbf{R}-\Omega_{p} t\right)}$, which we denote by $\rho_{1 e}^{\prime}(\mathbf{R}, t)$.

Before proceeding, we note that in this Appendix all summations over momentum states have been converted to an integration over a continuum of states via the standard substitution $\sum_{\mathbf{p}} \rightarrow \frac{V}{(2 \pi \hbar)^{3}} \int d^{3} p$. The coefficient $\rho_{1 e}^{\prime}$ is related to the coherence in position space, $\rho_{1 e}^{\prime}(\mathbf{R}, t)$, and the momentum space density matrix elements, $\rho_{1 e}\left(\mathbf{p}, \mathbf{p}^{\prime} ; t\right)=\rho_{1 e}^{\prime}\left(\mathbf{p}, \mathbf{p}^{\prime} ; t\right) e^{i \Omega_{p} t}$, by

$$
\begin{aligned}
\rho_{1 e}^{\prime}(\mathbf{R}, t) & =\rho_{1 e}^{\prime} e^{-i\left(\mathbf{k}_{p} \cdot \mathbf{R}-\Omega_{p} t\right)} \\
& =\frac{1}{(2 \pi \hbar)^{3}} \iint d^{3} p d^{3} p^{\prime} \rho_{1 e}^{\prime}\left(\mathbf{p}, \mathbf{p}^{\prime} ; t\right) e^{i \Omega_{p} t} e^{i\left(\mathbf{p}-\mathbf{p}^{\prime}\right) \cdot \mathbf{R} / \hbar} \delta\left(\mathbf{p}-\mathbf{p}^{\prime}+\hbar \mathbf{k}_{p}\right)
\end{aligned}
$$

The coherence, $\rho_{1 e}^{\prime}\left(\mathbf{p}, \mathbf{p}^{\prime} ; t\right) e^{i \Omega_{p} t}$, has been written in the Schrödinger representation and is obtained from the density matrix for the atom plus pump fields by tracing over the number of photons in the pump fields,

$$
\rho_{1 e}^{\prime}\left(\mathbf{p}, \mathbf{p}^{\prime} ; t\right)=e^{-i \Omega_{p} t} e^{-i \omega_{1 e} t} \sum_{n_{1}, n_{2}} \rho_{1 e}^{I}\left(\mathbf{p}, n_{1}, n_{2} ; \mathbf{p}^{\prime}, n_{1}, n_{2} ; t\right)
$$

where $\rho_{1 e}^{I}\left(\mathbf{p}, n_{1}, n_{2} ; \mathbf{p}^{\prime}, n_{1}^{\prime}, n_{2}^{\prime} ; t\right)$ is in the interaction representation with respect to the internal energy levels and pump fields. One cannot derive a differential equation for $\rho_{1 e}^{\prime}\left(\mathbf{p}, \mathbf{p}^{\prime} ; t\right)$ starting from the original Hamiltonian in Eq. (3a) since the excited state has been adiabatically eliminated from the effective Hamiltonian. However, by reintroducing an interaction term

$$
H_{a f}=\hbar\left[g_{1}|e\rangle\left\langle 1\left|e^{i \mathbf{k}_{1} \cdot \mathbf{R}}+g_{2}\right| e\right\rangle\left\langle 2\left|e^{i \mathbf{k}_{2} \cdot \mathbf{R}}+\chi_{p}\right| e\right\rangle\langle 1| e^{i\left(\mathbf{k}_{1} \cdot \mathbf{R}-\Omega_{p} t\right)}\right]+\text { h.c. }
$$


into the Hamiltonian and writing

$$
\dot{\rho}_{1 e}^{\prime}\left(\mathbf{p}, \mathbf{p}^{\prime} ; t\right)=\frac{\partial}{\partial t}\left(e^{-i \Omega_{p} t} e^{-i \omega_{1 e} t} \sum_{n_{1}, n_{2}} \rho_{1 e}^{I}\left(\mathbf{p}, n_{1}, n_{2} ; \mathbf{p}^{\prime}, n_{1}, n_{2} ; t\right)\right) .
$$

one finds the equation of motion for $\rho_{1 e}^{I}\left(\mathbf{p}, n_{1}, n_{2} ; \mathbf{p}^{\prime}, n_{1}^{\prime}, n_{2}^{\prime}\right)$ to be

$$
\begin{aligned}
\dot{\rho}_{1 e}^{I}\left(\mathbf{p}, n_{1}, n_{2} ; \mathbf{p}^{\prime}, n_{1}^{\prime}, n_{2}^{\prime}\right)= & {\left[-i \omega_{\mathbf{p} \mathbf{p}^{\prime}}-\left(\gamma+\gamma_{e}\right) / 2\right] \rho_{1 e}^{I}\left(\mathbf{p}, n_{1}, n_{2} ; \mathbf{p}^{\prime}, n_{1}^{\prime}, n_{2}^{\prime}\right) } \\
& -i \chi_{1}^{*} e^{i\left(\Omega_{1}-\omega_{e 1}\right) t}\left[\rho_{e e}^{I}\left(\mathbf{p}+\hbar \mathbf{k}_{1}, n_{1}-1, n_{2} ; \mathbf{p}^{\prime}, n_{1}^{\prime}, n_{2}^{\prime}\right)\right. \\
& \left.-\rho_{11}^{I}\left(\mathbf{p}, n_{1}, n_{2} ; \mathbf{p}^{\prime}-\hbar \mathbf{k}_{1}, n_{1}^{\prime}+1, n_{2}^{\prime}\right)\right] \\
& -i \chi_{p}^{*} e^{i\left(\Omega_{p}-\omega_{e 1}\right) t}\left[\rho_{e e}^{I}\left(\mathbf{p}+\hbar \mathbf{k}_{p}, n_{1}, n_{2} ; \mathbf{p}^{\prime}, n_{1}^{\prime}, n_{2}^{\prime}\right)\right. \\
& \left.-\rho_{11}^{I}\left(\mathbf{p}, n_{1}, n_{2} ; \mathbf{p}^{\prime}-\hbar \mathbf{k}_{p}, n_{1}^{\prime}, n_{2}^{\prime}\right)\right] \\
& +i \chi_{2}^{*} e^{i\left(\Omega_{2}+\omega_{2 e}\right) t} \rho_{12}^{I}\left(\mathbf{p}, n_{1}, n_{2} ; \mathbf{p}^{\prime}-\hbar \mathbf{k}_{2}, n_{1}^{\prime}, n_{2}^{\prime}+1\right),
\end{aligned}
$$

where the $t$ argument has been dropped. By carrying out the trace in Eq. (37), one obtains terms such as,

$$
\rho_{11}^{I}\left(\mathbf{p} ; \mathbf{p}^{\prime}-\hbar \mathbf{k}_{1}\right)=\sum_{n_{1}, n_{2}} \rho_{11}^{I}\left(\mathbf{p}, n_{1}, n_{2} ; \mathbf{p}^{\prime}-\hbar \mathbf{k}_{1}, n_{1}+1, n_{2}\right)
$$

so that the equation of motion for $\rho_{1 e}^{\prime}\left(\mathbf{p} ; \mathbf{p}^{\prime}\right)$ has a form which is identical to that which would have been obtained using classical pump fields,

$$
\begin{aligned}
\dot{\rho}_{1 e}^{\prime}\left(\mathbf{p} ; \mathbf{p}^{\prime}\right)= & -\left[i\left(\Omega_{p}+\omega_{1 e}+\omega_{\mathbf{p p}^{\prime}}\right)+\left(\gamma+\gamma_{e}\right) / 2\right] \rho_{1 e}^{\prime}\left(\mathbf{p} ; \mathbf{p}^{\prime}\right) \\
& -i \chi_{p}^{*}\left[\rho_{e e}^{I}\left(\mathbf{p}+\hbar \mathbf{k}_{p} ; \mathbf{p}^{\prime}\right)-\rho_{11}^{I}\left(\mathbf{p} ; \mathbf{p}^{\prime}-\hbar \mathbf{k}_{p}\right)\right] \\
& -i \chi_{1}^{*} e^{-i \delta_{p 1} t}\left[\rho_{e e}^{I}\left(\mathbf{p}+\hbar \mathbf{k}_{1} ; \mathbf{p}^{\prime}\right)-\rho_{11}^{I}\left(\mathbf{p} ; \mathbf{p}^{\prime}-\hbar \mathbf{k}_{1}\right)\right] \\
& +i \chi_{2}^{*} e^{-i \delta_{p 2} t} \rho_{12}^{I}\left(\mathbf{p} ; \mathbf{p}^{\prime}-\hbar \mathbf{k}_{2}\right) .
\end{aligned}
$$

In terms of the perturbation series solution

$$
\begin{aligned}
& \rho_{11}^{I}\left(\mathbf{p} ; \mathbf{p}^{\prime}\right)=\rho_{11}^{(0)}\left(\mathbf{p} ; \mathbf{p}^{\prime}\right)+\rho_{11}^{+}\left(\mathbf{p} ; \mathbf{p}^{\prime}\right) e^{i \delta_{p 1} t}+\rho_{11}^{-}\left(\mathbf{p} ; \mathbf{p}^{\prime}\right) e^{-i \delta_{p 1} t} \\
& \rho_{12}^{I}\left(\mathbf{p} ; \mathbf{p}^{\prime}\right)=\left[\rho_{12}^{(0)}\left(\mathbf{p} ; \mathbf{p}^{\prime}\right)+\rho_{12}^{+}\left(\mathbf{p} ; \mathbf{p}^{\prime}\right) e^{i \delta_{p 1} t}+\rho_{12}^{-}\left(\mathbf{p} ; \mathbf{p}^{\prime}\right) e^{-i \delta_{p 1} t}\right] e^{i \delta_{12} t}
\end{aligned}
$$


where $\rho_{j j^{\prime}}^{(0)}\left(\mathbf{p} ; \mathbf{p}^{\prime}\right)$ are independent of $\chi_{p}$ and $\rho_{j j^{\prime}}^{ \pm}\left(\mathbf{p} ; \mathbf{p}^{\prime}\right)$ are linear in $\chi_{p}$, the steady state solution for large detuning is

$$
\rho_{1 e}^{\prime}\left(\mathbf{p} ; \mathbf{p}^{\prime}\right) \approx \frac{1}{\Delta}\left(\chi_{2}^{*} \rho_{12}^{+}\left(\mathbf{p} ; \mathbf{p}^{\prime}-\hbar \mathbf{k}_{2}\right)+\chi_{1}^{*} \rho_{11}^{+}\left(\mathbf{p} ; \mathbf{p}^{\prime}-\hbar \mathbf{k}_{1}\right)+\chi_{p}^{*} \rho_{11}^{(0)}\left(\mathbf{p} ; \mathbf{p}^{\prime}-\hbar \mathbf{k}_{p}\right)\right)
$$

By making a change of variables, $\mathbf{p}-\mathbf{p}^{\prime} \rightarrow \mathbf{p}-\mathbf{p}^{\prime}-\hbar \mathbf{k}_{p}$ in Eq. (36), one gets,

$$
\begin{aligned}
\rho_{1 e}^{\prime}(\mathbf{R}, t)= & \frac{e^{-i\left(\mathbf{k}_{p} \cdot \mathbf{R}-\Omega_{p} t\right)}}{(2 \pi \hbar)^{3} \Delta} \iint d^{3} p d^{3} p^{\prime} e^{i\left(\mathbf{p}-\mathbf{p}^{\prime}\right) \cdot \mathbf{R} / \hbar} \delta\left(\mathbf{p}-\mathbf{p}^{\prime}\right) \\
& \times\left\{\chi_{2}^{*}\left(\rho_{12}^{+}\left(\mathbf{p} ; \mathbf{p}^{\prime}+\hbar \mathbf{k}_{p 2}\right)+\rho_{12}^{+}\left(\mathbf{p}-\hbar \mathbf{k}_{p 1} ; \mathbf{p}^{\prime}+\hbar \mathbf{k}_{12}\right)\right)+\right. \\
& \left.\chi_{1}^{*}\left(\rho_{11}^{+}\left(\mathbf{p} ; \mathbf{p}^{\prime}+\hbar \mathbf{k}_{p 1}\right)+\rho_{11}^{+}\left(\mathbf{p}-\hbar \mathbf{k}_{p 1} ; \mathbf{p}^{\prime}\right)\right)+\chi_{p}^{*} \rho_{11}^{(0)}\left(\mathbf{p} ; \mathbf{p}^{\prime}\right)\right\}
\end{aligned}
$$

One must now obtain equations for $\rho_{11}^{I}\left(\mathbf{p} ; \mathbf{p}^{\prime}\right)$ and $\rho_{12}^{I}\left(\mathbf{p} ; \mathbf{p}^{\prime}\right)$ using the effective Hamiltonian $\mathbf{H}_{t o t}=\mathbf{H}+\mathbf{V}_{I}$, solve these equations, and then extract $\rho_{12}^{+}\left(\mathbf{p} ; \mathbf{p}^{\prime}\right)$ and $\rho_{11}^{+}\left(\mathbf{p} ; \mathbf{p}^{\prime}\right)$ from these solutions using Eqs. (41a-41b).

The terms appearing in Eq. (43) may be expressed in terms of the dressed state density matrix elements. If one is interested only in terms linear in $\chi_{p}$, one can expand the dressed state density matrix to first order in $\chi_{p}$ as

$$
\begin{aligned}
\rho_{I I^{\prime}} & =\left[\rho_{I I^{\prime}}^{(0)}+\rho_{I I^{\prime}}^{+} e^{i\left(\Omega_{p}-\omega_{10}\right) t}+\rho_{I I^{\prime}}^{-} e^{-i\left(\Omega_{p}-\omega_{10}\right) t}\right] e^{i \omega_{10} t} ; \\
\rho_{J J^{\prime}} & =\left[\rho_{J J^{\prime}}^{(0)}+\rho_{J J^{\prime}}^{+} e^{i\left(\Omega_{p}+\omega_{20}\right) t}+\rho_{J J^{\prime}}^{-} e^{-i\left(\Omega_{p}+\omega_{20}\right) t}\right] e^{i \omega_{20} t} ;
\end{aligned}
$$

where $I, J=\left\{A_{0}, B_{0}\right\}, I^{\prime}=\left\{A_{1}, B_{1}\right\}$, and $J^{\prime}=\left\{A_{2}, B_{2}\right\}$. The $\rho_{i j}^{+}\left(\mathbf{p} ; \mathbf{p}^{\prime}\right)$ needed in Eq. (43) can be expressed in terms of dressed state density matrix elements using Eqs. (21,22, 49) as 


$$
\begin{aligned}
\rho_{11}^{+}\left(\mathbf{p} ; \mathbf{p}+\hbar \mathbf{k}_{p 1}\right)= & \sin \theta_{0}(\mathbf{p}) \sin \theta_{1}(\mathbf{p}) \rho_{A_{0} A_{1}}^{+}-\sin \theta_{0}(\mathbf{p}) \cos \theta_{1}(\mathbf{p}) \rho_{A_{0} B_{1}}^{+} \\
& -\cos \theta_{0}(\mathbf{p}) \sin \theta_{1}(\mathbf{p}) \rho_{B_{0} A_{1}}^{+}+\cos \theta_{0}(\mathbf{p}) \cos \theta_{1}(\mathbf{p}) \rho_{B_{0} B_{1}}^{+} ; \\
\rho_{11}^{+}\left(\mathbf{p}-\hbar \mathbf{k}_{p 1} ; \mathbf{p}\right)= & \sin \theta_{2}(\mathbf{p}) \sin \theta_{0}(\mathbf{p}) \rho_{A_{2} A_{0}}^{+}-\sin \theta_{2}(\mathbf{p}) \cos \theta_{0}(\mathbf{p}) \rho_{A_{2} B_{0}}^{+} \\
& -\cos \theta_{2}(\mathbf{p}) \sin \theta_{0}(\mathbf{p}) \rho_{B_{2} A_{0}}^{+}+\cos \theta_{2}(\mathbf{p}) \cos \theta_{0}(\mathbf{p}) \rho_{B_{2} B_{0}}^{+} ; \\
\rho_{12}^{+}\left(\mathbf{p} ; \mathbf{p}+\hbar \mathbf{k}_{p 2}\right)= & e^{i \phi_{d}}\left(-\sin \theta_{0}(\mathbf{p}) \cos \theta_{1}(\mathbf{p}) \rho_{A_{0} A_{1}}^{+}-\sin \theta_{0}(\mathbf{p}) \sin \theta_{1}(\mathbf{p}) \rho_{A_{0} B_{1}}^{+}\right. \\
& \left.+\cos \theta_{0}(\mathbf{p}) \cos \theta_{1}(\mathbf{p}) \rho_{B_{0} A_{1}}^{+}+\cos \theta_{0}(\mathbf{p}) \sin \theta_{1}(\mathbf{p}) \rho_{B_{0} B_{1}}^{+}\right) ; \\
\rho_{12}^{+}\left(\mathbf{p}-\hbar \mathbf{k}_{p 1} ; \mathbf{p}+\hbar \mathbf{k}_{12}\right)= & e^{i \phi_{d}}\left(-\sin \theta_{2}(\mathbf{p}) \cos \theta_{0}(\mathbf{p}) \rho_{A_{2} A_{0}}^{+}-\sin \theta_{2}(\mathbf{p}) \sin \theta_{0}(\mathbf{p}) \rho_{A_{2} B_{0}}^{+}\right. \\
& \left.+\cos \theta_{2}(\mathbf{p}) \cos \theta_{0}(\mathbf{p}) \rho_{B_{2} A_{0}}^{+}+\cos \theta_{2}(\mathbf{p}) \sin \theta_{0}(\mathbf{p}) \rho_{B_{2} B_{0}}^{+}\right) .
\end{aligned}
$$

The state vector in the Schrödinger representation may be expanded in terms of the dressed states for the 0,1 , and 2 manifolds,

$\left|\Psi>=c_{A_{0}}^{s}(\mathbf{p})\right| A_{0}>+c_{B_{0}}^{s}(\mathbf{p})\left|B_{0}>+c_{A_{1}}^{s}(\mathbf{p})\right| A_{1}>+c_{B_{1}}^{s}(\mathbf{p})\left|B_{1}>+c_{A_{2}}^{s}(\mathbf{p})\right| A_{2}>+c_{B_{2}}^{s}(\mathbf{p}) \mid B_{2}>$.

In the following, the momentum labels are suppressed. The Schrödinger equation for the dressed state amplitudes is then given by

$$
i \hbar \dot{\mathbf{c}}=\left(\mathbf{H}_{o}+\mathbf{V}_{I d}\right) \mathbf{c}
$$

where

$$
\mathbf{H}_{o}=\operatorname{diag}\left(E_{A}^{(1)}, E_{B}^{(1)}, E_{A}^{(0)}, E_{B}^{(0)}, E_{A}^{(2)}, E_{B}^{(2)}\right)
$$

and matrix elements of $\mathbf{V}_{I d}$ are given in Eqs. (33a $33 \mathrm{~h}$ ).

Using Eq. (46) along with the ground state decay rate $\gamma$ and incoherent pumping to the 0 manifold, one finds that density matrix elements for the six dressed states in the three manifolds (0), (1), (2), evolve as

$$
\left(\frac{d}{d t}+\gamma\right) \rho_{d}=\frac{1}{i \hbar}\left[\mathbf{H}_{o}+\mathbf{V}_{I d}, \rho_{d}\right]+\Lambda_{D}
$$


where

$$
\rho_{d}\left(\mathbf{p}, \mathbf{p}^{\prime}\right)=\mathbf{c}(\mathbf{p}) \mathbf{c}^{\dagger}\left(\mathbf{p}^{\prime}\right)=\left(\begin{array}{cccccc}
\rho_{A_{1} A_{1}} & \rho_{A_{1} B_{1}} & \rho_{A_{1} A_{0}} & \rho_{A_{1} B_{o}} & \rho_{A_{1} A_{2}} & \rho_{A_{1} B_{2}} \\
\rho_{B_{1} A_{1}} & \rho_{B_{1} B_{1}} & \rho_{B_{1} A_{0}} & \rho_{B_{1} B_{o}} & \rho_{B_{1} A_{2}} & \rho_{B_{1} B_{2}} \\
\rho_{A_{0} A_{1}} & \rho_{A_{0} B_{1}} & \rho_{A_{0} A_{0}} & \rho_{A_{0} B_{o}} & \rho_{A_{0} A_{2}} & \rho_{A_{0} B_{2}} \\
\rho_{B_{0} A_{1}} & \rho_{B_{0} B_{1}} & \rho_{B_{0} A_{0}} & \rho_{B_{0} B_{o}} & \rho_{B_{0} A_{2}} & \rho_{B_{0} B_{2}} \\
\rho_{A_{2} A_{1}} & \rho_{A_{2} B_{1}} & \rho_{A_{2} A_{0}} & \rho_{A_{2} B_{o}} & \rho_{A_{2} A_{2}} & \rho_{A_{2} B_{2}} \\
\rho_{B_{2} A_{1}} & \rho_{B_{2} B_{1}} & \rho_{B_{2} A_{0}} & \rho_{B_{2} B_{o}} & \rho_{B_{2} A_{2}} & \rho_{B_{2} B_{2}}
\end{array}\right)
$$

and the pumping matrix, $\Lambda_{d}$, has the block diagonal form

$$
\Lambda_{D}=\operatorname{diag}\left(\mathbf{0}, \Lambda_{d}\left(\mathbf{p}, \mathbf{p}^{\prime}\right), \mathbf{0}\right)
$$

where $\Lambda_{d}(\mathbf{p})$ has the basic structure given in Eq. (24), modified to allow for $\mathbf{p}, \mathbf{p}^{\prime}$ coherence. In particular, the off diagonal elements of $\Lambda_{d}\left(\mathbf{p}, \mathbf{p}^{\prime}\right)$ that give rise to the nonsecular contribution to the line shape are of the form

$\Lambda_{A B}\left(\mathbf{p}, \mathbf{p}^{\prime}+\hbar \mathbf{k}_{12}\right)=\Lambda_{2}\left(\mathbf{p}+\hbar \mathbf{k}_{12}, \mathbf{p}^{\prime}+\hbar \mathbf{k}_{12}\right) \cos \left[\theta_{0}(\mathbf{p})\right] \sin \left[\theta_{0}\left(\mathbf{p}^{\prime}\right)\right]-\Lambda_{1}\left(\mathbf{p}, \mathbf{p}^{\prime}\right) \sin \left[\theta_{0}(\mathbf{p})\right] \cos \left[\theta_{0}\left(\mathbf{p}^{\prime}\right)\right]$

The general form of the solution is linked to the incoherent pumping of levels 1 and 2. For a subrecoil cooled vapor, the pumping rate density for bare state density matrix elements $\rho_{i j}\left(\mathbf{p} ; \mathbf{p}^{\prime}\right)$ is assumed to be

$$
\Lambda_{i j}\left(\mathbf{p}, \mathbf{p}^{\prime}\right)=\Lambda_{i} V^{-1}(2 \pi \hbar)^{3} \delta(\mathbf{p}) \delta\left(\mathbf{p}-\mathbf{p}^{\prime}\right) \delta_{i j}
$$

where $\Lambda_{i} V^{-1}$ has the dimensions of (volume $\times$ time $)^{-1}$ and can be interpreted as the pumping rate to state 1 or 2 in position space. With this form of pumping, $\rho_{i j}^{I}\left(\mathbf{p} ; \mathbf{p}^{\prime}\right)$, must be proportional to $\delta\left(\mathbf{p}-\mathbf{p}^{\prime}-\hbar \mathbf{k}^{\prime}\right)$ where $\mathbf{k}^{\prime}$ is some algebraic combination of the pump and probe field propagation vectors. To obtain $\rho_{1 e}^{\prime}$ from Eq. (43), one must keep only those terms in the integrand of Eq. (43) proportional to $\delta\left(\mathbf{p}-\mathbf{p}^{\prime}\right)$. The $\rho_{11}^{(0)}(\mathbf{p} ; \mathbf{p})$ term in Eq. (43) makes no contribution to the absorption since it is real and will be ignored from this point on. 
The incoherent pumping of states 1 and 2 populate two different manifolds. The pumping of state 1 populates the $\left(\mathbf{p}=0, n_{1}, n_{2}\right)$ manifold while the pumping of state 2 populates the $\left(\mathbf{p}=-\hbar \mathbf{k}_{12}, n_{1}, n_{2}\right)$ manifold since this manifold involves state 2 with zero momentum. Thus in viewing absorption or emission, two distinct initial state manifolds must be included, leading to the possibility of sixteen rather than eight components of the spectrum. Here we set $\Lambda_{2}=0$.

Substituting Eqs. (45a-45d) into Eq. (43) and using the steady state solutions for $\rho_{I I^{\prime}}^{+}$ and $\rho_{J J^{\prime}}^{+}$obtained from Eqs. (44a-44b) and Eq. (48), one finds, after some manipulation, the final expression for the absorption coefficient,

$$
\begin{aligned}
& \left(\frac{\alpha}{\alpha_{0}}\right)=\left(\frac{\alpha}{\alpha_{0}}\right)_{\sec }+\left(\frac{\alpha}{\alpha_{0}}\right)_{n s} \\
& \left(\frac{\alpha}{\alpha_{0}}\right)_{n s}=\frac{|G|}{|\Delta|} \frac{\Lambda_{A B}}{\gamma} \frac{\sin 2 \theta_{0}}{2\left(\left(\omega_{A B}^{(0)}\right)^{2}+\gamma^{2}\right)}\left[-\left(\psi \eta \sin \theta_{1}-\frac{1}{\eta} \cos \theta_{1}\right)^{2}\left(\Gamma_{A_{0} A_{1}}\left(\Delta^{\prime}\right)+\Gamma_{B_{0} A_{1}}\left(\Delta^{\prime}\right)\right)\right. \\
& -\left(\psi \eta \cos \theta_{1}+\frac{1}{\eta} \sin \theta_{1}\right)^{2}\left(\Gamma_{A_{0} B_{1}}\left(\Delta^{\prime}\right)+\Gamma_{B_{0} B_{1}}\left(\Delta^{\prime}\right)\right) \\
& +\left(\eta^{2}-\eta^{-2}-2 \psi \cot 2 \theta_{0}\right)\left(\sin ^{2} \theta_{2}\left(\Gamma_{A_{0} A_{2}}\left(\Delta^{\prime}\right)+\Gamma_{B_{0} A_{2}}\left(\Delta^{\prime}\right)\right)+\cos ^{2} \theta_{2}\left(\Gamma_{A_{0} B_{2}}\left(\Delta^{\prime}\right)+\Gamma_{B_{0} B_{2}}\left(\Delta^{\prime}\right)\right)\right) \\
& \Gamma_{A_{0} A_{1}}\left(\Delta^{\prime}\right)=\left[\left(\Delta^{\prime}-\omega_{\hbar \mathbf{k}_{p 1}}-\frac{1}{2}\left(\omega_{A B}^{(0)}-\omega_{A B}^{(1)}\right)\right) \omega_{A B}^{(0)}+\gamma^{2}\right] L_{A_{0} A_{1}}\left(\Delta^{\prime}\right) \\
& \Gamma_{B_{0} A_{1}}\left(\Delta^{\prime}\right)=\left[-\left(\Delta^{\prime}-\omega_{\hbar \mathbf{k}_{p 1}}+\frac{1}{2}\left(\omega_{A B}^{(0)}+\omega_{A B}^{(1)}\right)\right) \omega_{A B}^{(0)}+\gamma^{2}\right] L_{B_{0} A_{1}}\left(\Delta^{\prime}\right) ; \\
& \Gamma_{A_{0} B_{1}}\left(\Delta^{\prime}\right)=\left[\left(\Delta^{\prime}-\omega_{\hbar \mathbf{k}_{p 1}}-\frac{1}{2}\left(\omega_{A B}^{(0)}+\omega_{A B}^{(1)}\right)\right) \omega_{A B}^{(0)}+\gamma^{2}\right] L_{A_{0} B_{1}}\left(\Delta^{\prime}\right) \\
& \Gamma_{B_{0} B_{1}}\left(\Delta^{\prime}\right)=\left[-\left(\Delta^{\prime}-\omega_{\hbar \mathbf{k}_{p 1}}+\frac{1}{2}\left(\omega_{A B}^{(0)}-\omega_{A B}^{(1)}\right)\right) \omega_{A B}^{(0)}+\gamma^{2}\right] L_{B_{0} B_{1}}\left(\Delta^{\prime}\right) \text {; } \\
& \Gamma_{A_{0} A_{2}}\left(\Delta^{\prime}\right)=\left[-\left(\Delta^{\prime}+\omega_{\hbar \mathbf{k}_{p 1}}+\frac{1}{2}\left(\omega_{A B}^{(0)}-\omega_{A B}^{(2)}\right)\right) \omega_{A B}^{(0)}+\gamma^{2}\right] L_{A_{0} A_{2}}\left(\Delta^{\prime}\right) \text {; } \\
& \Gamma_{B_{0} A_{2}}\left(\Delta^{\prime}\right)=\left[\left(\Delta^{\prime}+\omega_{\hbar \mathbf{k}_{p 1}}-\frac{1}{2}\left(\omega_{A B}^{(0)}+\omega_{A B}^{(2)}\right)\right) \omega_{A B}^{(0)}+\gamma^{2}\right] L_{B_{0} A_{2}}\left(\Delta^{\prime}\right) ; \\
& \Gamma_{A_{0} B_{2}}\left(\Delta^{\prime}\right)=\left[-\left(\Delta^{\prime}+\omega_{\hbar \mathbf{k}_{p 1}}+\frac{1}{2}\left(\omega_{A B}^{(0)}+\omega_{A B}^{(2)}\right)\right) \omega_{A B}^{(0)}+\gamma^{2}\right] L_{A_{0} B_{2}}\left(\Delta^{\prime}\right) \text {; } \\
& \Gamma_{B_{0} B_{2}}\left(\Delta^{\prime}\right)=\left[\left(\Delta^{\prime}+\omega_{\hbar \mathbf{k}_{p 1}}-\frac{1}{2}\left(\omega_{A B}^{(0)}-\omega_{A B}^{(2)}\right)\right) \omega_{A B}^{(0)}+\gamma^{2}\right] L_{B_{0} B_{2}}\left(\Delta^{\prime}\right)
\end{aligned}
$$


to the fact that the secular approximation consists solely of neglecting the off-diagonal components of $\Lambda_{d}$. Since the first order solutions, $\rho_{I I^{\prime}}^{+}$and $\rho_{J J^{\prime}}^{+}$, are linear in the pumping terms, $\left(\frac{\alpha}{\alpha_{0}}\right)_{\text {sec }}$ contain terms proportional to $\Lambda_{A}$ and $\Lambda_{B}$ while $\left(\frac{\alpha}{\alpha_{0}}\right)_{n s}$ is proportional to $\Lambda_{A B}$. This simplification would not occur for a more complex decay scheme for states $\mid 1>$ and $\mid 2>$ since the decay would couple density matrix elements in a field dependent manner (see [23]).

The nonsecular term, $\left(\frac{\alpha}{\alpha_{0}}\right)_{n s}$, consist of dispersion-like structures centered at the same locations as the resonances in $\left(\frac{\alpha}{\alpha_{0}}\right)_{\text {sec }}$. In the secular limit, $\left(\frac{\alpha}{\alpha_{0}}\right)_{n s} \ll\left(\frac{\alpha}{\alpha_{0}}\right)_{\sec }$ and $\left(\frac{\alpha}{\alpha_{0}}\right)_{n s}$ can usually be ignored. Notice that if one chooses $\psi$ and $\eta$ such that a pair of absorption lines in $\left(\frac{\alpha}{\alpha_{0}}\right)_{\text {sec }}$ vanish, then the corresponding terms in $\left(\frac{\alpha}{\alpha_{0}}\right)_{n s}$ also vanish so that $\left(\frac{\alpha}{\alpha_{0}}\right)$ is identically zero. However, this will not be true for the gain terms in $\left(\frac{\alpha}{\alpha_{0}}\right)_{\text {sec }}$ since the corresponding terms in $\left(\frac{\alpha}{\alpha_{0}}\right)_{n s}$ have a different interference coefficient, $\left(\eta^{2}-\eta^{-2}-2 \psi \cot 2 \theta_{0}\right)$. Figure 6 shows a plot of the non-secular absorption coefficient for the same parameters as Fig. 3. In this plot, the non-secular terms are $\sim 1000$ times smaller than the secular terms. 


\section{REFERENCES}

[1] J. Guo, P.R. Berman, B. Dubetsky, and G. Grynberg, Phys. Rev. A 46, 1426 (1992).

[2] J. Guo and P.R. Berman, Phys. Rev. A 47, 4128 (1993).

[3] P.R. Berman, B. Dubetsky, and J.Guo, Phys. Rev. A 51, 3947 (1995).

[4] B. Dubetsky and P.R. Berman, Phys. Rev. A 52, R2519 (1995).

[5] J.Y. Courtois, G. Grynberg, B. Lounis, and P. Verkerk, Phys. Rev. Lett. 72, 3017 (1994).

[6] S. Guibal, C. Triche, L. Guidoni, P. Verkerk, and G. Grynberg, Opt. Commun. 131, 61 (1996).

[7] D.R. Meacher, D. Boiron, H. Metcalf, C. Saloman, and G. Grynberg, Phys. Rev. A 50, R1992 (1994).

[8] M. Kozuma, Y. Imai, N. Nakagawa, and M. Ohtsu, Phys. Rev. A 52, R3421 (1995); M. Kozuma, N. Nakagawa, W. Jhe, and M. Ohtsu, Phys. Rev. Lett. 76, 2428 (1996).

[9] D.M. Stamper-Kurn, A. P. Chikkatur, A. Görlitz, S. Inouye, S. Gupta, D. E. Pritchard, and W. Ketterle, Phys. Rev. Lett. 83, 2876 (1999).

[10] J. Stenger, S. Inouye, A. P. Chikkatur, D. M. Stamper-Kurn, D. E. Pritchard, and W. Ketterle, Phys. Rev. Lett. 82, 4569 (1999).

[11] M. G. Raizen, J. Koga, B. Sundaram, Y. Kishimoto, H. Takuma, and T. Tajima, Phys. Rev. 58, 4757 (1998).

[12] See, for example, R. Bonifacio and L. De Salvo, Nucl. Instrum. Methods A 341, 360 (1994); R. Bonifacio, L. De Salvo, L. M. Narducci, and E. J. D’Angelo, Phys. Rev. A 50, 1716 (1994); R. Bonifacio and L. De Salvo, Opt. Commun. 115, 505 (1995); L. De Salvo, R. Cannerozzi, R. Bonifacio, E. J. D’Angelo and L. M. Narducci, Phys. Rev. A 52, 2342 (1995); R. Bonifacio, L. De Salvo, L. M. Narducci, and E. J. D’Angelo, 
in Coherent and collective interactions of particles and radiation beams, edited by A. Aspect, W. A. Barletta and R. Bonifacio (IOS Press, Amsterdam, 1996).

[13] M. G. Moore and P Meystre, Phys. Rev. A 59, R1754 (1999).

[14] P.R. Berman and B. Dubetsky, Phys. Rev A 62, 053412 (2000).

[15] The dressed states defined here differ by the interchange of $\mid 1>$ and $\mid 2>$ with respect to the dressed states defined in [13].

[16] The initial state manifold only is incoherently pumped, although a distribution of intial state manifolds dictated by $\Lambda(\mathbf{p})$ is permitted. The final state manifold involves states having different quantum numbers for the pump fields and different center-of-mass momenta.

[17] B. Dubetsky and P. R. Berman , Phys. rev. A 56, 1091 (1997).

[18] See, for example, L. V. Hau, S. E. Harris, Z. Dutton, and C. H. Behroozi, Nature 397, 594 (1999); M. M. Kash, V. A. Sautenkov, A. S. Zibrov, L. Hollberg, G. W. Welch, M. D. Lukin, Y. Rostovtsev, E. S. Fry, and M. O. Scully, Phys. Rev. Lett. 82, 5229 (1999); D. Budker, D. F. Kimball, S. M. Rochester, and V. V. Yashchuk, Phys. Rev. Lett. 83, 1767 (1999).

[19] S. Inouye, A. P. Chikkatur, D. M. Stamper-Kurn, J. Stenger, D. E. Pritchard, and W. Ketterle, Science 285, 571 (1999).

[20] M. G. Moore and P. Meystre, Phys. Rev. Let. 83, 5202 (1999).

[21] M. R. Mathews, D. S. Hall, D. S. Jin, J. R. Ensher, C. E. Wieman, E. A. Cornell, F. Dalfovo, C. Minniti, and S. Stringari, Phys. Rev. Lett. 81, 243 (1998).

[22] D. M. Stamper-Kurn, M. R. Andrews, A. P. Chikkatur, S. Inouye, H.-J. Miesner, J. Stenger, and W. Ketterle, Phys. Rev. Lett. 80, 2027 (1998).

[23] P. R. Berman, Phys. Rev. A 53, 2627 (1996). 
Figure 1. Schematic diagram of atom-field system.

Figure 2. (a) Transitions between the 0 manifold and the 1 and 2 manifolds leading to probe gain or absorption in the bare state picture. The states in the 1 and 2 manifolds are displaced from the states in the 0 manifold by an amount $\sim \hbar \Omega_{1}$. The Rabi frequencies shown are those which couple the states from the 0 manifold to the 1 and 2 manifolds. (b) Energy levels in the dressed state basis. The center of the 1 manifold has an energy $\hbar \omega_{10}$ above the center of the 0 manifold and similarly, the center of the 2 manifold is $\hbar \omega_{20}$ below the 0 manifold.(c) Illustration of the coupling of the pump and probe fields to the dressed states for the $\left|A_{0}>\rightarrow\right| A_{1}>$ transition. The Rabi frequencies shown are those that couple the bare state $\mid 1>$ component of $\mid A_{0}>$ to the bare state components of $\mid A_{1}>$.

Figure 3. Plot of $\left(\frac{\alpha}{\alpha_{0}}\right)_{\mathrm{sec}}$ for $\Lambda_{2}=0, \Lambda_{1} / \gamma=1, \psi=-1$ and $\eta=2$ showing all eight absorption and emission lines. The detuning is $\delta_{12} / \gamma=300$ and the two-photon pump Rabi frequency is $|G| / \gamma=250$. The recoil energies are $\omega_{\hbar \mathbf{k}_{12}} / \gamma=40, \omega_{\hbar \mathbf{k}_{p 1}} / \gamma=60$, and $\hbar \mathbf{k}_{p 1} \cdot \mathbf{k}_{12} / M \gamma=80$. Note that $\gamma \ll \gamma_{e}$ where $\gamma_{e}$ is the excited state decay rate.

Figure 4. Plot of $\left(\frac{\alpha}{\alpha_{0}}\right)_{\mathrm{sec}}$ showing destructive interference. Parameters are the same as Fig. 4 except for $\psi$ and $\eta$. The solid line corresponds to $\psi=-1$ and $\eta=\sqrt{\tan \theta_{1}}=0.8383$ while the dotted line corresponds to $\psi=+1$ and $\eta=\sqrt{\cot \theta_{1}}=1.1928$.

Figure 5. Plot showing sixteen lines in the $\left(\frac{\alpha}{\alpha_{0}}\right)_{\text {sec }}$ probe spectrum for $\Lambda_{2} / \gamma=\Lambda_{1} / \gamma=1$, $\psi=+1$ and $\eta=0.1$. The detuning is $\delta_{12} / \gamma=500$ and the two-photon pump Rabi frequency is $|G| / \gamma=750$. The recoil energies are $\omega_{\hbar \mathbf{k}_{12}} / \gamma=50, \omega_{\hbar \mathbf{k}_{p 1}} / \gamma=75$, and $\hbar \mathbf{k}_{p 1} \cdot \mathbf{k}_{12} / M \gamma=-50$.

Figure 6. Plot of $\left(\frac{\alpha}{\alpha_{0}}\right)_{n s}$ for the same parameters as Fig. (3). The non-secular absorption coefficient has dispersionlike structures at the same location is the line centers of $\left(\frac{\alpha}{\alpha_{0}}\right)_{\sec }$. The amplitudes of these non-secular terms is typically $~ 1000$ times smaller than the secular line strengths, consistent with $\gamma / \omega_{A B}^{(0)}=0.00177$. 


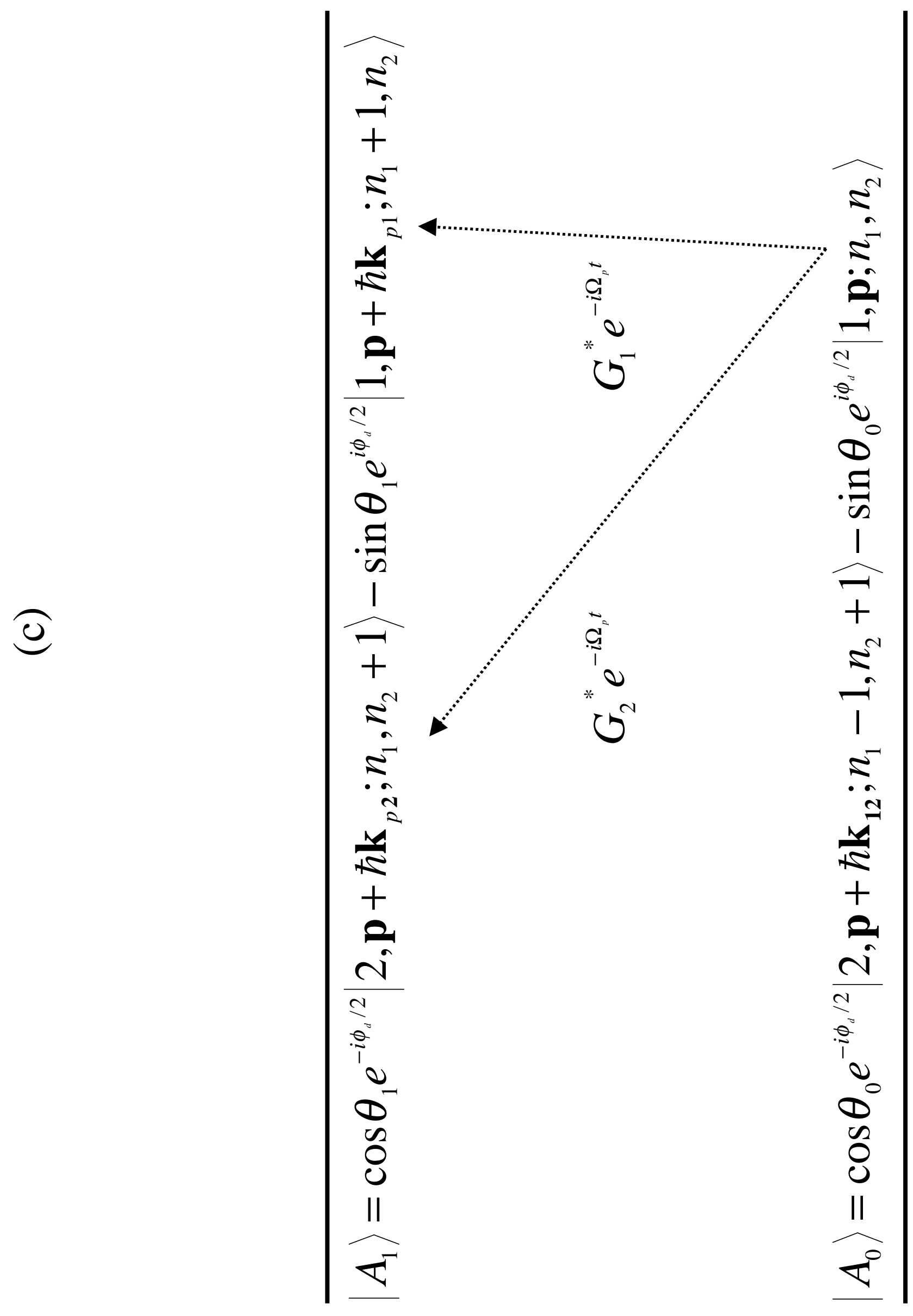

